\title{
Targeted neoadjuvant therapy in the HER-2-positive breast cancer patients: a systematic review and meta-analysis
}

This article was published in the following Dove Press journal:

OncoTargets and Therapy

\author{
Wenhua Ma ${ }^{1, *}$ \\ Fugang Zhao ${ }^{2, *}$ \\ Changpeng Zhou' \\ Yongqian Zhang' \\ Yingchun Zhao' \\ $\mathrm{Na} \mathrm{Li}{ }^{\prime}$ \\ Peng $\mathrm{Xie}^{3}$
}

'Department of Oncology, The First Hospital of Hebei Medical University, Shijiazhuang 050000, Hebei Province, China; ${ }^{2}$ Department of Traditional

Chinese Medicine, The First Hospital of Hebei Medical University, Shijiazhuang 050000, Hebei Province, China; ${ }^{3}$ Department of Nuclear Medicine, The Third Hospital of Hebei Medical University, Shijiazhuang 050000, Hebei Province, China

*These authors contributed equally to this work
Correspondence: Peng Xie Department of Nuclear Medicine, The Third Hospital of Hebei Medical University, No I 39 Zi Qiang Road, Shijiazhuang 050000, Hebei Province, China

Tel +86 I85 33II 2767

Email woxinfly1982@।26.com
Aim: To evaluate efficacy and safety of lapatinib or trastuzumab alone or both plus chemotherapy for the treatment of breast cancer patients with positive HER-2 expression.

Methods: Cochrane Central Register of Controlled Trials, PubMed, MEDLINE, OVID, Embase, Chinese Biomedical Literature Database, and China Academic Journals Database were searched from 1994 through December 2017 using the keywords "breast cancer", "preoperative”, "neoadjuvant", "lapatinib", "pertuzumab", "Herceptin", and "trastuzumab".

Results: Meta-analysis found that pathological complete response (PCR; risk ratio $[R R]=0.82$, 95\% CI: 0.72-0.93) and tall PCR (tPCR; RR=0.77, 95\% CI: 0.67-0.88) of chemotherapy plus lapatinib were significantly less effective or safe compared to that of chemotherapy plus trastuzumab $(P<0.05)$. PCR $(\mathrm{RR}=1.30,95 \% \mathrm{CI}: 1.15-1.47)$ and $\mathrm{PCR}(\mathrm{RR}=1.32,95 \% \mathrm{CI}$ : 1.16-1.50) of chemotherapy plus both lapatinib and trastuzumab were significantly superior to that of chemotherapy plus trastuzumab alone $(P<0.05)$. However, there was no significant difference in breast reservation rate between chemotherapy plus lapatinib vs chemotherapy plus trastuzumab ( $\mathrm{RR}=0.91,95 \% \mathrm{CI}$ : $0.72-1.16)$ or chemotherapy plus both lapatinib and trastuzumab $(\mathrm{RR}=1.11,95 \% \mathrm{CI}: 0.73-1.68, P>0.05)$. Incidence of diarrhea, hepatic toxicity, and skin rash in the groups of chemotherapy plus lapatinib or chemotherapy plus both lapatinib and trastuzumab was significantly higher than that in chemotherapy plus trastuzumab $(P<0.05)$.

Conclusion: Efficacy of lapatinib was less than that of trastuzumab, but incidence of adverse effect of lapatinib was higher than that of trastuzumab. Combination of chemotherapy plus both lapatinib and trastuzumab could significantly increase PCR and PCR in breast cancer patients, but rate of breast conservation, event-free survival, and overall survival was not significantly improved. Incidence of diarrhea, hepatic toxicity, and skin rash was significantly increased in the groups using lapatinib.

Keywords: breast cancer, neoadjuvant, lapatinib, trastuzumab, HER-2-positive

\section{Introduction}

Neoadjuvant approach of breast cancer has been endorsed by several groups and experts for a wide variety of reasons. ${ }^{1}$ For instance, neoadjuvant treatment could reverse late phase breast cancer that could not be surgically removed into the cancer, which might be treated by surgery with increasing rate of breast reservation. It could also increase the sensitivity of the tumor to systemic therapy, and thus, it is currently the preferred treatment for locally advanced breast cancer. ${ }^{2,3}$

HER-2 (ErbB2) is a member of the ErbB family of receptors. Overexpression of HER-2 in human tumors is closely associated with increased angiogenesis and expression of vascular endothelial growth factor. ${ }^{4}$ It has been reported that $20 \%-25 \%$ 
of breast cancers were HER-2 positive. ${ }^{5}$ Trastuzumab is a humanized murine monoclonal antibody that inhibits ligandindependent HER-2 and HER-3 signaling, ${ }^{6}$ and triggers antibody-dependent cellular cytotoxicity. ${ }^{7}$ Trastuzumab has been approved for clinical use in $1998,{ }^{8}$ and increasing reports of evidence-based medicine indicated that trastuzumab is effective in the treatment of breast cancer at various stages including early stage and stages II and III breast cancer. ${ }^{8-11}$ Specifically, trastuzumab in combination with neoadjuvant chemotherapy followed by adjuvant trastuzumab monotherapy has been recommended for the treatment of breast cancer patients with positive HER-2 since 2011 . $^{12}$

Lapatinib is a reversible, dual EGFR (HER-1)/HER-2 tyrosine kinase inhibitor (TKI). ${ }^{13}$ Lapatinib (with capecitabine) has been suggested for the treatment of HER-2positive breast cancer patients, whose disease has progressed during previous trastuzumab-based therapy. ${ }^{14}$ This systematic review and meta-analysis were, therefore, designed to evaluate the outcomes of efficacy and safety for chemotherapy plus lapatinib, chemotherapy plus trastuzumab, or chemotherapy plus both lapatinib and trastuzumab in the treatment of breast cancer.

\section{Methods}

\section{Inclusion criteria}

Randomized clinical trials (RCT) on breast cancer treatment in women at age 18 or older, with histologically proven stages I, II, III or inflammatory breast cancer. Patients with positive HER-2 expression were determined by immunohistochemistry or fluorescence in situ hybridization; patients with adequate cardiac function had baseline left ventricular ejection fraction of $\geq 50 \%$.

\section{Intervention methods}

Control group: chemotherapy plus trastuzumab. Study groups: chemotherapy plus lapatinib or chemotherapy plus lapatinib and trastuzumab.

\section{End points of the study}

Pathological complete response (PCR) is defined as absence of invasive tumor cells in the breast. Tall PCR (tPCR) is defined as no invasive cancer in the breast and no pathological involvement axillary lymph node, which was analyzed based on hormone receptor (HR) status. Adverse events with III-IV grade include nausea, vomiting, diarrhea, hepatic toxicity, left ventricular ejection fraction (LVEF) decline, skin rash, and fatigue. The adverse effects were evaluated following the National Cancer Institute's Common Terminology Criteria for Adverse Events.

\section{Literature searching strategy}

The following databases were searched: Cochrane Central Register of Controlled Trials, PubMed, MEDLINE, OVID, Embase, Chinese Biomedical Literature Database, and China Academic Journals Database. The following keywords were used to search the literature from 1994 through January 2017: "breast cancer", "preoperative", "neoadjuvant", "lapatinib”, "pertuzumab", "Herceptin", and "trastuzumab".

\section{Data extraction}

All authors had been trained to understand purpose of systematic review and know the methods of meta-analysis. Two authors (Fu-Gang Zhao and Chang-Peng Zou) were primarily responsible to read through all titles and abstracts in order to exclude nonrelated literature. Full text was then obtained and selected for further data extraction. The following information was extracted: 1) general information: title, first author, country, language, funding, and extractor; 2) patient's information: age, gender, intervention reagents and protocol, dose of medication, duration of therapy, and follow-up; and 3 ) clinical outcomes.

\section{Evaluation on the quality of the studies}

This was performed following the Quality Evaluation Criteria recommended by Cochrane Manual, version 5.3. Specifically, quality of the studies was evaluated in the following six aspects: randomization, hidden assignment, blind study, data integrity, bias in data collection, and other potential bias. If a study met all of aforementioned criteria, it was at low risk of bias, and in contrast, if a study did not meet the criteria, it was considered as high risk of bias. If a study lacks of detail description, or unknown risk, or the relationship with the study was unclear, it indicated moderate risk. Data quality was assessed by two researchers (Yong-Qian Zhang and Ying-Chun Zhao). If there was a discrepancy, a third researcher $(\mathrm{Li} \mathrm{Na})$ did further assessment.

\section{Statistical analysis}

A statistical analysis software provided by Cochrane (Revman5.3) was used to perform statistical analysis. The data quality, analysis on the combined data, survival rate (event-free survival [EFS] and overall survival [OS]), hazard risk, relative risk, and its 95\% CI were analyzed by using this software. A fixed effect model was applied when no 
heterogeneity was observed among the studies. Alternatively, a random effect model was applied if the heterogeneity between studies was $P<0.10$ and $\mathrm{I}^{2}>50 \%$, which was considered as heterogeneous between the studies

\section{Results}

\section{Study selection and trial information}

As shown in Figure 1, based on the searching strategy, total 149 published studies were retrieved. Of them, 25 publications were in Chinese and 124 were in English. After excluding repeated publication and non-RCT studies, total ten studies were included in this systematic review and meta-analysis. ${ }^{15-24}$ Three the ten studies were NeoALTTO results. ${ }^{16,17,25}$ As shown in Table 1, first author's name, stage, study groups, chemotherapy regimen, number of cases, duration of anti-HER-2 treatment, and outcomes of the treatment were included in the table.

\section{Assessment on risk of bias}

All studies included in this review were multicenter and controlled clinical studies. Double blind was applied

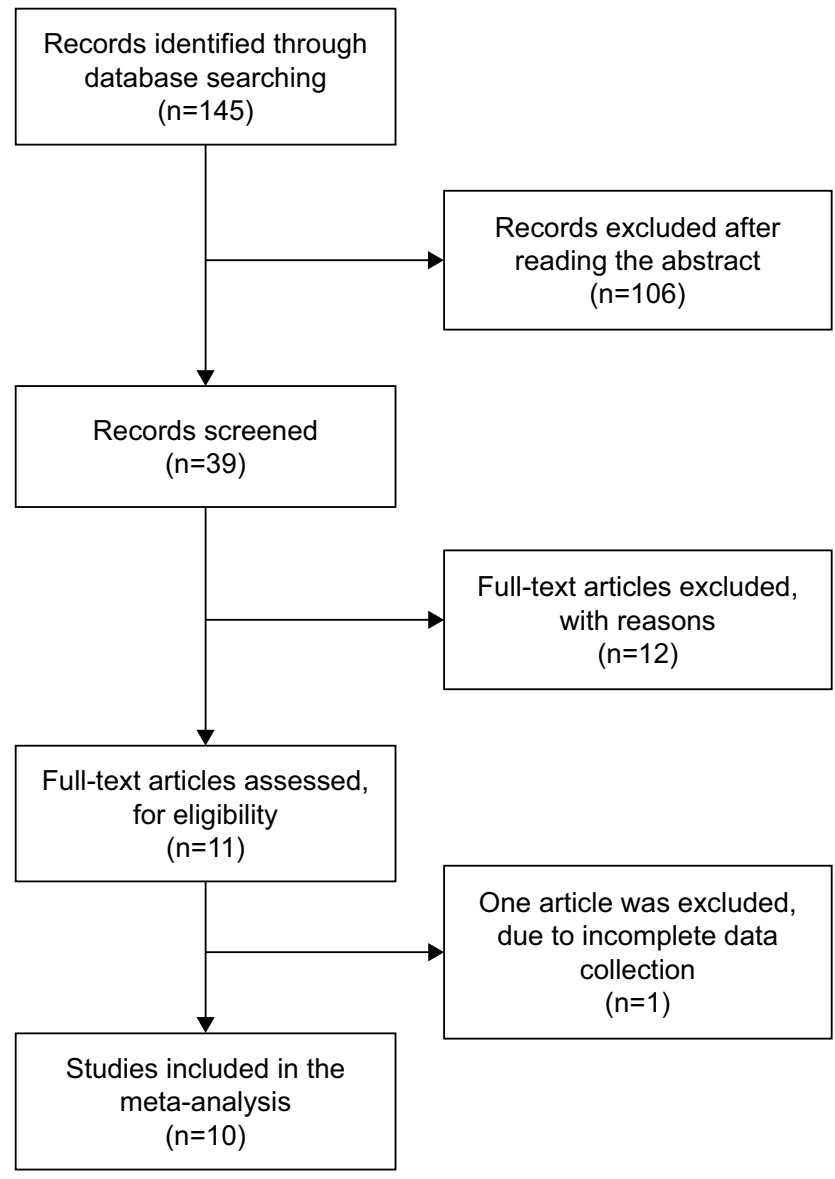

Figure I Flow chart of database search and literature selection. in assessing the results of the NeoALTTO study and GeparQuinto study. ${ }^{16-18,25}$ In the NeoALTTO study, only one paper ${ }^{25}$ was used for assessing the bias. There was report of drop-out in all of the studies and thus, intentionality analysis was performed. Randomization in grouping was used in all studies, and baseline balance was ensured in order to have comparability between the groups. In the CALGB 40601 study, group of chemotherapy plus lapatinib was closed earlier than expected. ${ }^{19}$ Assessment on the risk of bias outcome of each study was summarized in Figures 2 and 3.

\section{Meta-analysis results on patients' PCR and tPCR in breast and axillary lymph nodes}

Six of the ten studies compared the effect of chemotherapy plus lapatinib and chemotherapy plus trastuzumab on patients' PCR, ${ }^{18-22,25}$ while eight out of the ten studies compared the effect of the two treatments on patients' tPCR. ${ }^{18-25}$ It was found that chemotherapy plus trastuzumab on PCR $(\mathrm{RR}=0.82,95 \% \mathrm{CI}: 0.72-0.93)$ and $\mathrm{tPCR}(\mathrm{RR}=0.77,95 \% \mathrm{CI}$ : 0.67-0.88) were superior to chemotherapy plus lapatinib on PCR (RR=1.30, 95\% CI: 1.15-1.47, Figure 4) and tPCR $(\mathrm{RR}=1.32$, 95\% CI: 1.16-1.50, Figure 4).

Three studies analyzed effect of the treatment on PCR based on HER-2 positivity. ${ }^{22,23,25}$ Effect of chemotherapy plus trastuzumab $(\mathrm{RR}=0.69,95 \% \mathrm{CI}: 0.54-0.89)$, chemotherapy plus lapatinib ( $\mathrm{RR}=0.68$, 95\% CI: $0.53-0.87)$ or chemotherapy plus trastuzumab and lapatinib $(\mathrm{RR}=0.71,95 \% \mathrm{CI}$ : 0.59-0.85) on PCR was significantly better for the patients with negative HR expression than that for the patients with positive HR expression (Figure 5).

\section{Meta-analysis results on patients' breast conservation rate}

Four studies analyzed the effect of the treatment on breastconserving rate (BCS). ${ }^{17,18,20,23}$ The effect of chemotherapy plus lapatinib $(\mathrm{RR}=0.91,95 \% \mathrm{CI}: 0.72-1.16)$ or chemotherapy plus lapatinib and trastuzumab $(\mathrm{RR}=1.11,95 \% \mathrm{CI}$ : 0.73-1.68) was not significantly different from that of chemotherapy plus trastuzumab (Figure 6).

\section{Meta-analysis results on patients' EFS and OS rates}

One study reported the outcomes of the treatment on EFS and OS rates. ${ }^{16}$ EFS rate was not significantly different between the groups of chemotherapy plus lapatinib and chemotherapy 


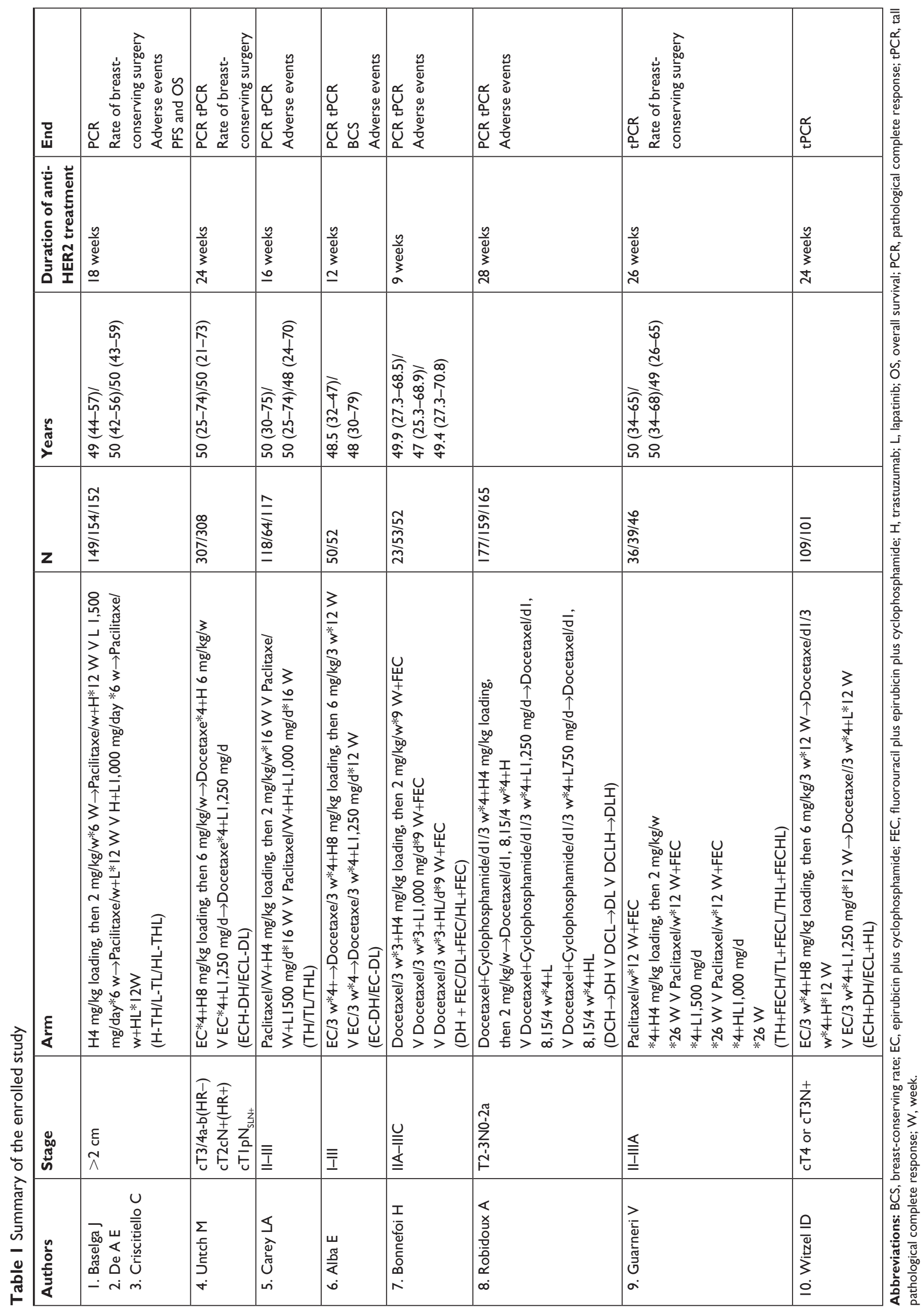




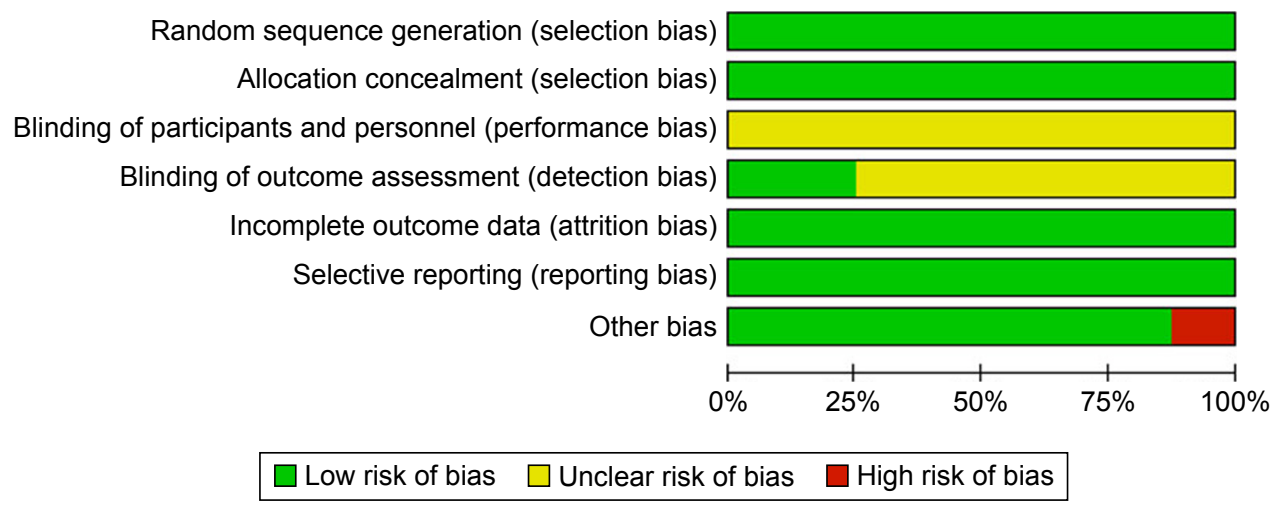

Figure 2 Risk of bias graph.

Note: Review of the authors' judgments about each risk of bias item was presented as percentages across all included studies.

plus trastuzumab (HR=1.06, 95\% CI: 0.66-1.69, Figure 7), or between the groups of chemotherapy plus lapatinib plus trastuzumab and chemotherapy plus trastuzumab alone (HR $=0.78,95 \%$ CI: 0.47-1.28, Figure 7).

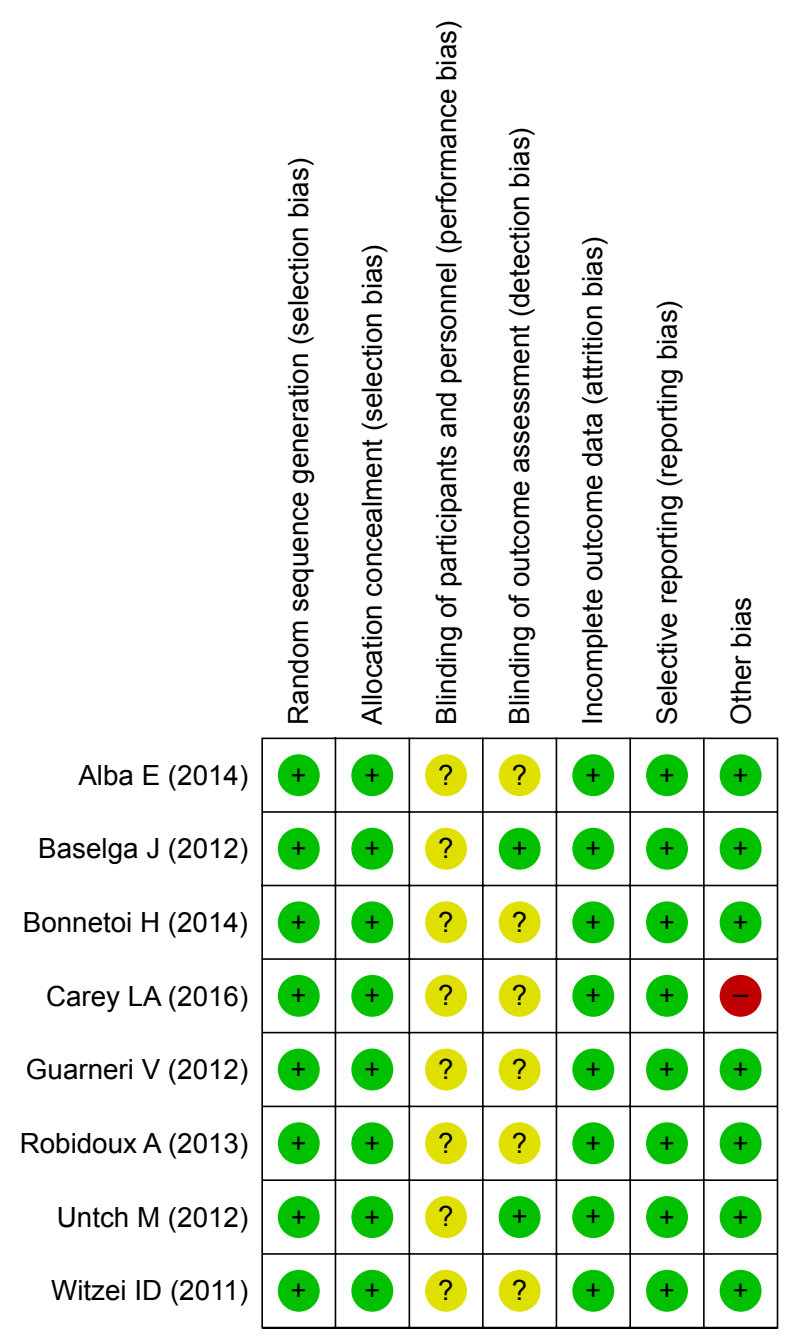

Figure 3 Risk of bias summary.

Note: Review of the authors' judgments about each risk of bias item for each literature was summarized.
Similarly, OS rate was not significantly different between the patients treated with lapatinib plus chemotherapy and trastuzumab plus chemotherapy (HR $=0.86,95 \% \mathrm{CI}$ : $0.45-1.63$, Figure 7) or compared with the patients treated with chemotherapy plus trastuzumab and trastuzumab alone ( $\mathrm{HR}=0.62$, 95\% CI: 0.30-1.25, Figure 7).

\section{Meta-analysis results on adverse effect}

Toxicity of the treatment was analyzed by comparing III-IV grade toxicity. Nausea ${ }^{18,21,22}$ was not significantly different in the groups of chemotherapy plus lapatinib $(\mathrm{RR}=0.98,95 \% \mathrm{CI}$ : 0.51-1.88) or chemotherapy plus lapatinib plus trastuzumab ( $R R=0.81,95 \%$ CI: $0.20-3.25)$ compared with the group of chemotherapy plus trastuzumab (Figure 7)..$^{21,22}$

Similarly, there were no significant differences between the groups in vomiting, LVEF decline or fatigue. Vomiting: $\mathrm{RR}=1.32$, 95\% CI: $0.58-2.97$ in comparison of chemotherapy plus lapatinib vs chemotherapy plus trastuzumab; ${ }^{21,22}$ $\mathrm{RR}=2.18,95 \%$ CI: $0.72-6.59$ in comparison of chemotherapy plus lapatinib plus trastuzumab vs chemotherapy plus trastuzumab. LVEF decline: $\mathrm{RR}=0.25,95 \% \mathrm{CI}$ : $0.03-2.22$ in comparison of chemotherapy plus lapatinib vs chemotherapy plus trastuzumab. ${ }^{18,23}$ Fatigue: RR=1.26, 95\% CI: $0.85-1.88$ in comparison of chemotherapy plus lapatinib ${ }^{18,20-23} \mathrm{vs}$ chemotherapy plus trastuzumab; ${ }^{21-23} \mathrm{RR}=0.84,95 \% \mathrm{CI}$ : $0.40-1.76$ in comparison of chemotherapy plus lapatinib plus trastuzumab vs chemotherapy plus trastuzumab.

However, diarrhea was significantly different between the groups of chemotherapy plus lapatinib ${ }^{18-20,23,25}$ or chemotherapy plus lapatinib plus trastuzumab compared to chemotherapy plus trastuzumab (RR=6.27, 95\% CI: 3.82-10.28; $\mathrm{RR}=8.70,95 \%$ CI: $4.45-17.01$, respectively). ${ }^{19,21,23,25}$

Similarly, hepatic toxicity and skin rash were also significantly different. Hepatic toxicity: $R R=2.03$, 95\% CI: $1.24-3.31$ in comparison of chemotherapy plus 
lapatinib $^{19-23,25}$ vs chemotherapy plus trastuzumab; ${ }^{19,21-23,25}$ $\mathrm{RR}=2.06,95 \% \mathrm{CI}: 1.19-3.54$ in comparison of chemotherapy plus lapatinib plus trastuzumab vs chemotherapy plus trastuzumab. Skin rash: $\mathrm{RR}=6.27,95 \% \mathrm{CI}: 3.84-10.28$ in comparison of chemotherapy plus lapatinib ${ }^{18,19,22}$ vs chemotherapy plus trastuzumab; ${ }^{19,22} \mathrm{RR}=8.70,95 \% \mathrm{CI}: 4.45-17.01$ in comparison of chemotherapy plus lapatinib plus trastuzumab vs chemotherapy plus trastuzumab.

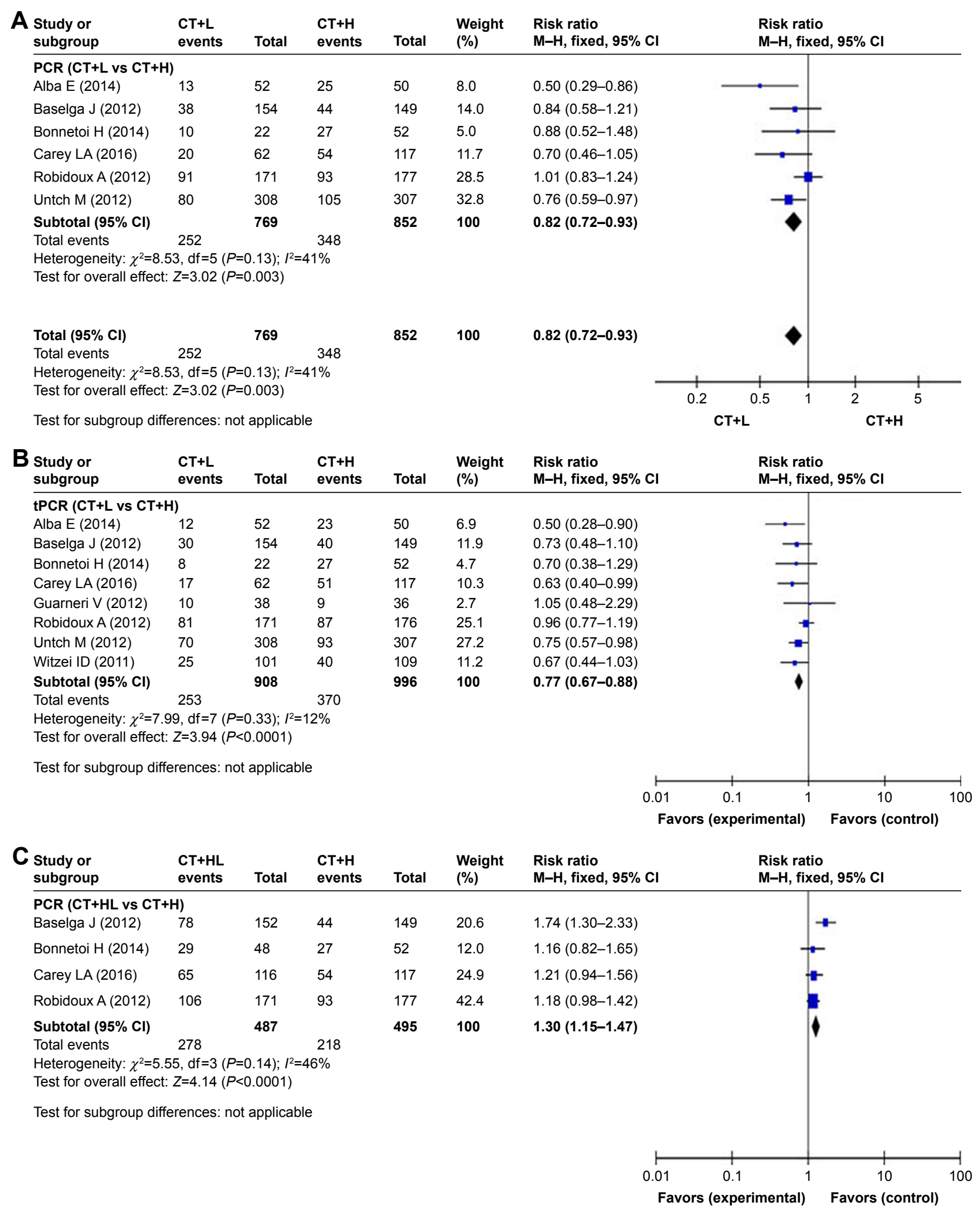




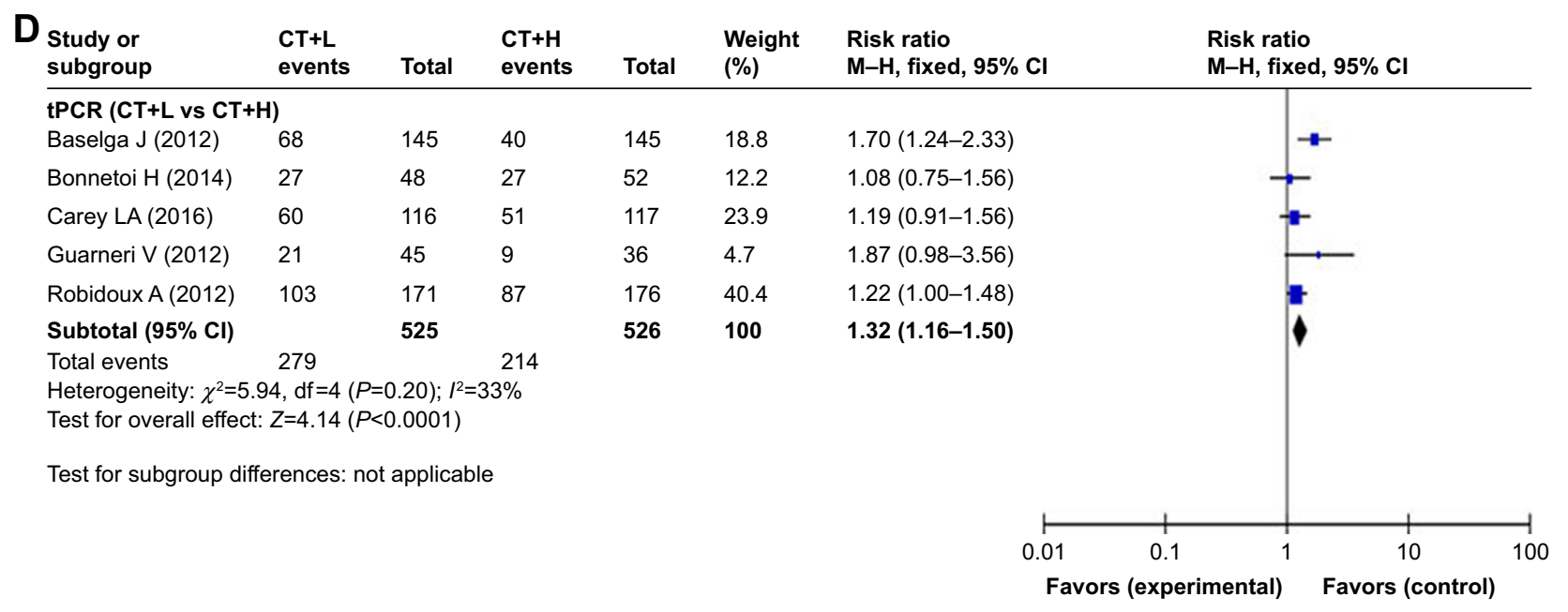

Figure 4 Forest plot for PCR and tPCR.

Notes: (A) $C T+L$ vs $C T+H$ for PCR. (B) $C T+L$ vs $C T+H$ for tPCR. (C) $C T+H L$ vs $C T+H$ for PCR. (D) $C T+H L$ vs $C T+H$ for $t P C R$.

Abbreviations: $\mathrm{PCR}$, pathological complete response; $\mathrm{PCCR}$, tall pathological complete response.

\section{Discussion}

In order to improve PCR rate and breast conservation rate by targeted and best combination of the HER-2 antagonists and chemotherapy, the current study performed systematic review and meta-analysis on the clinical trials of breast cancer treatment with lapatinib and/or trastuzumab plus chemotherapy. We found that chemotherapy plus trastuzumab is the best choice for the treatment of HER-2-positive breast adenocarcinoma. Specifically, we found that PCR and tPCR rates in the lapatinib group were lower than that in the trastuzumab group although breast conservation rate, 3-year EFS, and OS rates were not significantly different between the two groups. In addition, incidences of diarrhea, skin rash, and liver function damage were higher in the patients treated with lapatinib.

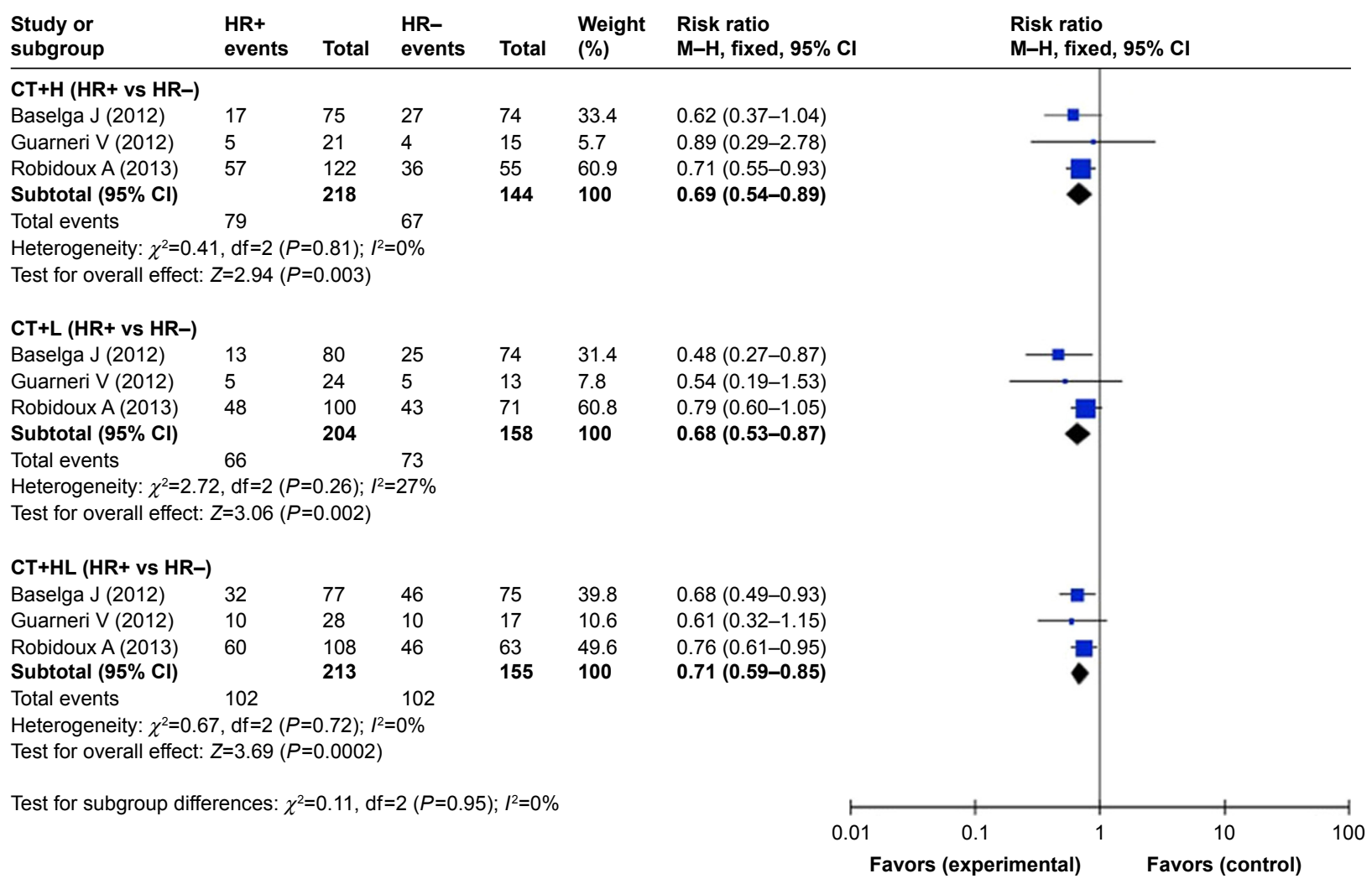

Figure 5 Forest plot by HER-2-positive $(H R+)$ vs HER-2-negative (HR-).

Abbreviation: HR, hormone receptor. 


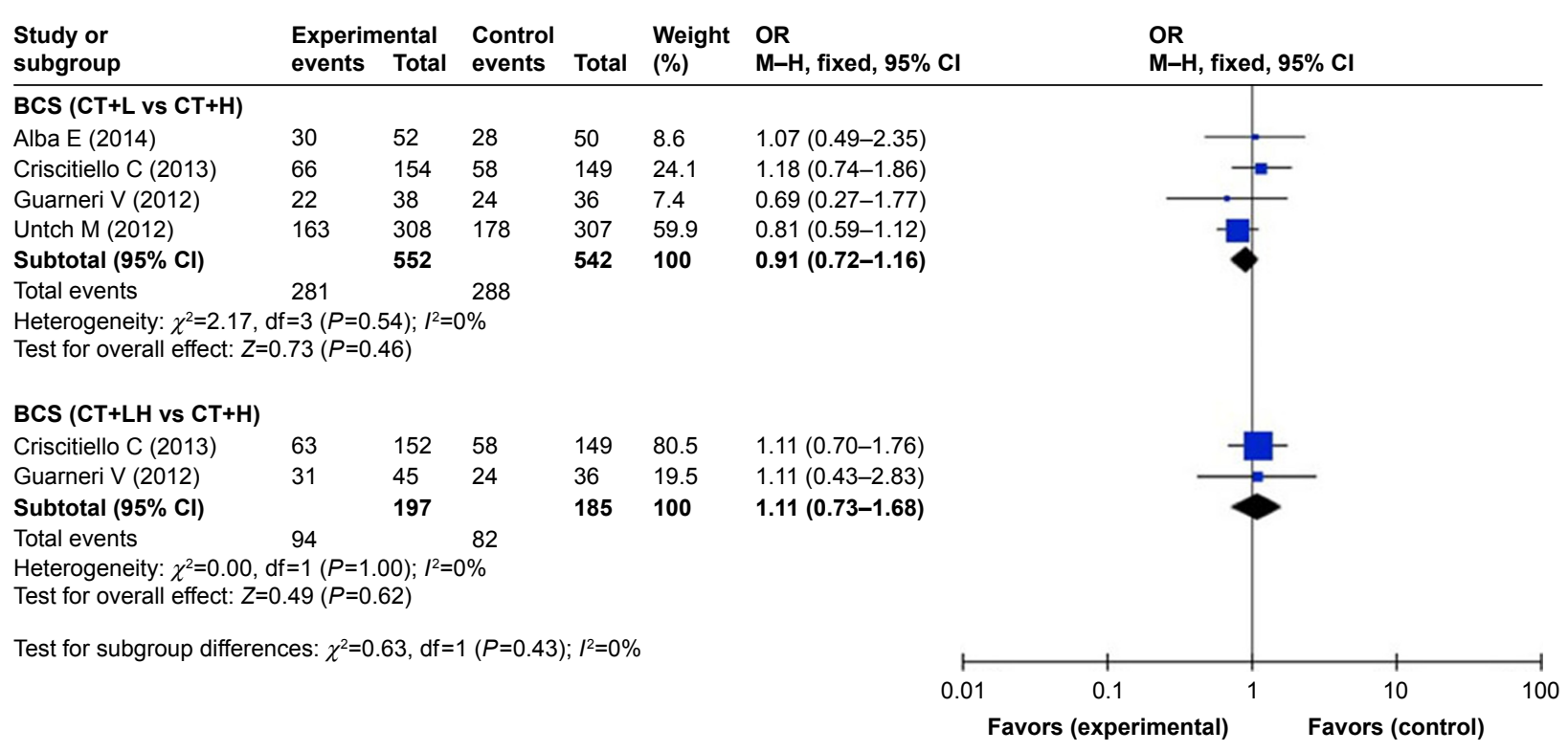

Figure 6 Forest plot for BCS comparison.

Abbreviation: $\mathrm{BCS}$, breast-conserving rate.

Lower PCR rates in the lapatinib group might be explained by a lower capability of the TKI, lapatinib, to block the HER-2 pathway compared to that by the antibody, trastuzumab. ${ }^{18}$ In contrast, trastuzumab may have additional antitumor effect by inducing an immune response via antibody-derived cellular cytotoxicity. ${ }^{7,18}$

Dual targeting on the HER-2-positive tumors by lapatinib and trastuzumab through their partially nonoverlapping mechanisms of action and the well-characterized synergistic interaction between them in the HER-2-positive breast cancer models have been reported. ${ }^{26-28}$ Specifically, lapatinib leads to an accumulation of HER-2 at the cell surface, and by which mechanism, it enhances trastuzumab-dependent (antibody-dependent) cellular cytotoxicity, ${ }^{29}$ and thus, dual HER-2-targeted therapies have been shown to improve outcomes for patients with HER-2-positive metastatic breast cancer. ${ }^{15,30}$ Consistently, the current systematic review and meta-analysis demonstrated that higher PCR was observed in the group of lapatinib plus trastuzumab in addition to the chemotherapy although breast conservation rate was not significantly improved. The neoadjuvant (TECHNO) study reported that chemotherapy plus trastuzumab resulted in significantly higher survival rate in PCR group compared to that of non-PCR group. ${ }^{31}$ Consistent with this report, the current meta-analysis revealed that combination of trastuzumab and lapatinib also resulted in higher survival rate in PCR group compared to the non-PCR group ( $\mathrm{HR}=0.32,95 \% \mathrm{CI}$ : $0.12-0.74, P=0.012$ ). However, OS rates in the three groups (chemotherapy plus lapatinib or trastuzumab or both) were not significantly different although targeted therapy could slightly increase PCR rate. Because usage of double targeted drugs in the developing countries means higher cost, we expect positive outcomes from the NSABP protocol B-41 in the comparisons of 5-year recurrence and OS among the treatment groups. ${ }^{22}$

Recently, studies on another set of dual targeting reagents on HER-2-positive tumors, that is, pertuzumab in combination with trastuzumab have been reported. Since trastuzumab is an antibody that targets subdomain IV in the extracellular region of HER-2, ${ }^{32}$ and pertuzumab is an antibody that targets the dimerization arm located in subdomain II of the extracellular region of HER-2,,$^{33}$ addition of pertuzumab to the regimen of trastuzumab and chemotherapy may provide an improvement in survival of HER-2-positive tumors. Results of clinical trials, however, indicated that outcomes of the combination may depend on the cell types of tumors. In this regard, von Minckwitz et al reported that addition of pertuzumab to a trastuzumab-containing adjuvant regimen moderately improved disease-free survival in women with breast cancer who were in poorest prognosis,${ }^{34}$ and Murthy et al reported that trastuzumab and pertuzumab-containing chemotherapy regimen yielded higher PCR rates in stage II-III HER-2-positive breast cancer patients compared to that trastuzumab plus chemotherapy regimen. ${ }^{35}$ In contrast, Tabernero et al reported that addition of pertuzumab to trastuzumab and chemotherapy did not significantly improve OS in patients with HER-2-positive metastatic gastric or gastroesophageal junction cancer compared with placebo. ${ }^{36}$ These findings suggested that studies on dual targeting on HER-2-positive tumors with pertuzumab and trastuzumab remains further investigated in different types of primary tumors. 


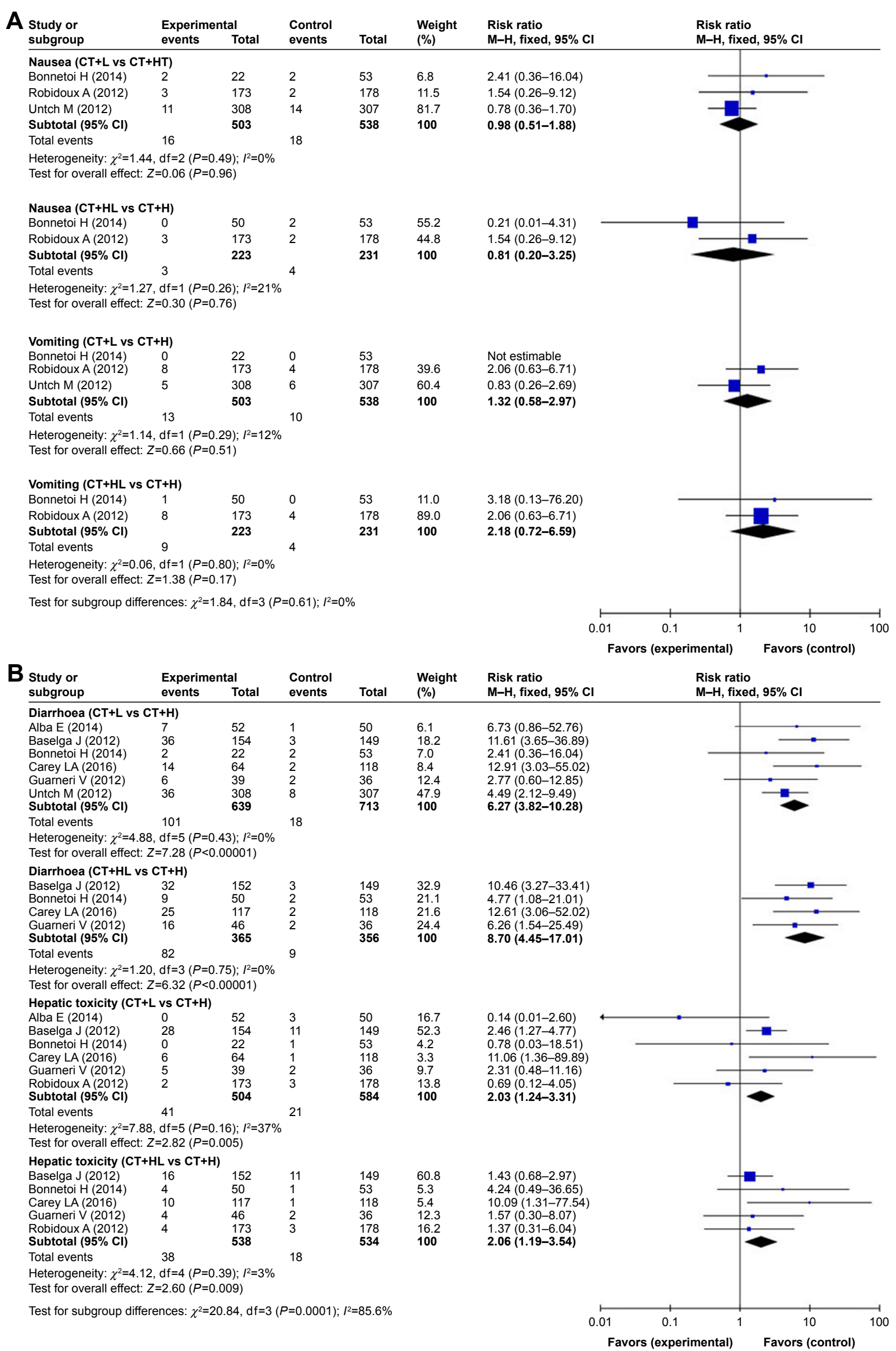

Figure 7 (Continued) 


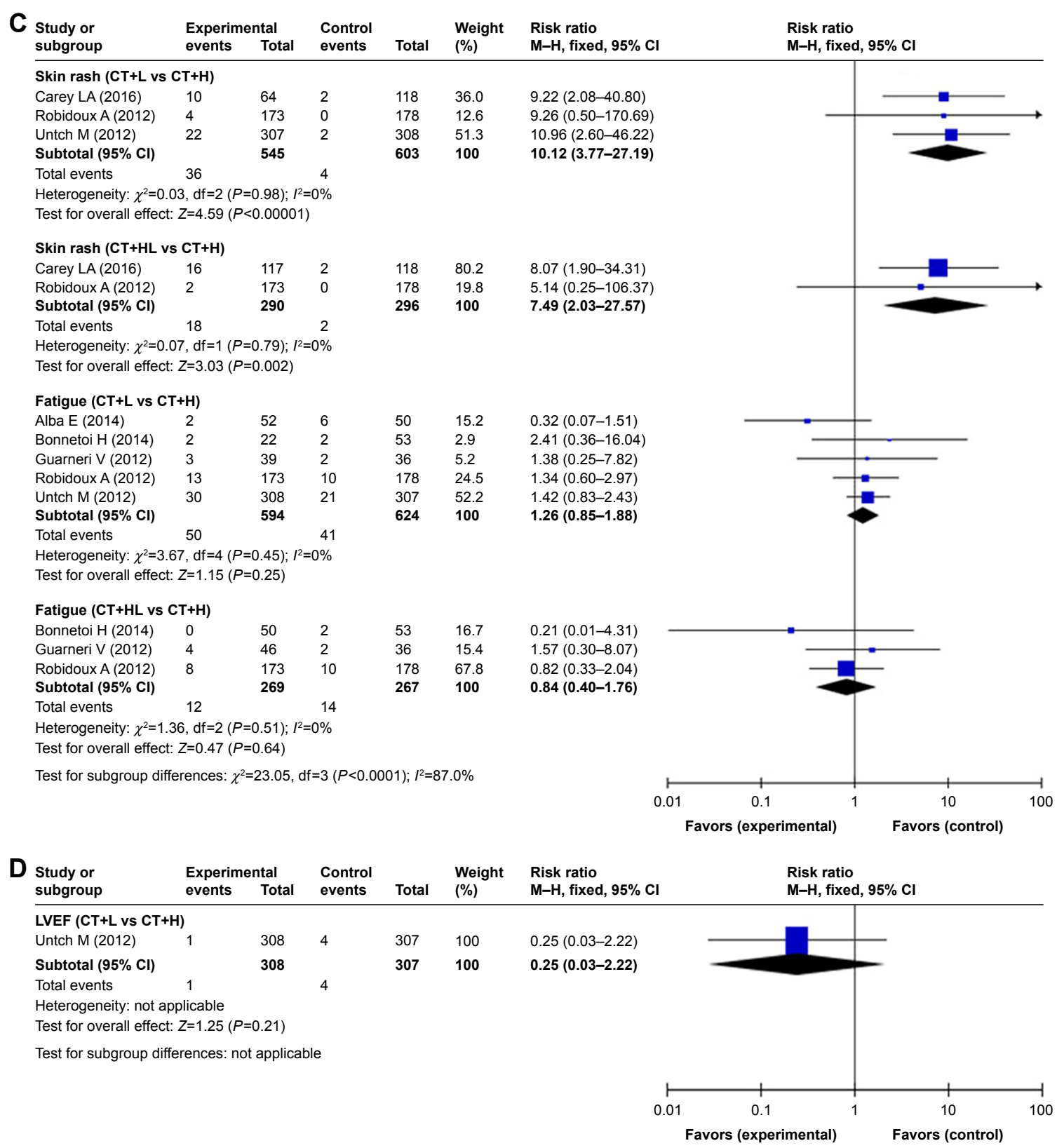

Figure 7 Forest plot for adverse effects comparison.

Notes: (A) Nausea and vomiting. (B) Diarrhea and hepatic toxicity. (C) Skin rash and fatigue. (D) LVEF comparison.

Abbreviation: LVEF, left ventricular ejection fraction.

Subgroup analysis indicated that hormone-receptornegative tumor had the greatest PCR. NeoALTTO study ${ }^{25}$ demonstrated that hormone-receptor-negative patients, who received combination of the targeted reagents, had the best outcome of 3-year EFS rate (86\%, 95\% CI: 75-92). Similarly, CTNeoBC study ${ }^{21}$ also demonstrated that the most favorable outcomes after PCR were recorded in HER2-positive, HR-negative patients who received trastuzumab (EFS: HR=0.15, 95\% CI: $0.09-0.27$; OS: $\mathrm{HR}=0.08,95 \%$ CI: $0.03-0.22$ ). These findings suggested that negative expression of hormone-receptor may predict promising outcomes.
The current analysis also found that application of lapatinib alone or in combination with trastuzumab resulted in more toxic side effects including diarrhea, skin rash, and liver function impairment, suggesting lapatinib may be associated with those toxic side effects. Due to $40 \%$ of toxic side effects in the combination group (double HER-2 blockade usage) in the NeoALTTO and NSABP protocol B-41 trials, neoadjuvant protocol therapy was discontinued in these trials..$^{22,25}$

\section{Conclusion}

Taken together, the current meta-analysis revealed that lapatinib caused higher occurrence rate of side effects, but 
lower rate of PCR and breast conservation in comparison to trastuzumab. When lapatinib was used in combination with trastuzumab, neither OS rate nor breast conservation rate was improved, although the combination did increase PCR or tPCR rate. These findings indicated that lapatinib is not recommended as single anti-HER-2-treatment in combination with chemotherapy and that combination of lapatinib with trastuzumab was not superior to that of trastuzumab alone.

\section{Disclosure}

The authors report no conflicts of interest in this work.

\section{References}

1. Kaufmann M, von Minckwitz G, Mamounas EP, et al. Recommendations from an international consensus conference on the current status and future of neoadjuvant systemic therapy in primary breast cancer. Ann Surg Oncol. 2012;19(5):1508-1516.

2. Ahmed MI, Lennard TW. Breast cancer: role of neoadjuvant therapy. Int J Surg. 2009;7(5):416-420.

3. Fisher B, Brown A, Mamounas E, et al. Effect of preoperative chemotherapy on local-regional disease in women with operable breast cancer: findings from National Surgical Adjuvant Breast and Bowel Project B-18. J Clin Oncol. 1997;15(7):2483-2493.

4. Kumar R, Yarmand-Bagheri R. The role of HER2 in angiogenesis. Semin Oncol. 2001;28(5 Suppl 16):27-32.

5. Nabholtz JM, Reese DM, Lindsay MA, Riva A. HER2-positive breast cancer: update on Breast Cancer International Research Group trials. Clin Breast Cancer. 2002;3(Suppl 2):S75-S79.

6. Junttila TT, Akita RW, Parsons K, et al. Ligand-independent HER2/ HER3/PI3K complex is disrupted by trastuzumab and is effectively inhibited by the PI3K inhibitor GDC-0941. Cancer Cell. 2009;15(5):429-440.

7. Barok M, Isola J, Pályi-Krekk Z, et al. Trastuzumab causes antibodydependent cellular cytotoxicity-mediated growth inhibition of submacroscopic JIMT-1 breast cancer xenografts despite intrinsic drug resistance. Mol Cancer Ther. 2007;6(7):2065-2072.

8. Slamon DJ, Leyland-Jones B, Shak S, et al. Use of chemotherapy plus a monoclonal antibody against HER2 for metastatic breast cancer that overexpresses HER2. N Engl J Med. 2001;344(11):783-792.

9. Davari M, Amani B, Mokarian F, Hoseini M, Akbarzadeh A, Heidarzadeh Khoramabadi N. Effectiveness of trastuzumab as adjuvant therapy in patients with early stage breast cancer: a systematic review and meta-analysis. Med J Islam Repub Iran. 2017;31:88-520.

10. García García-Esquinas MA, Arrazola García J, García-Sáenz JA, et al. Predictive value of PET-CT for pathological response in stages II and III breast cancer patients following neoadjuvant chemotherapy with docetaxel. Rev Esp Med Nucl Imagen Mol. 2014;33(1):14-21.

11. Gonzalez-Angulo AM, Hortobágyi GN, Esteva FJ. Adjuvant therapy with trastuzumab for HER-2/neu-positive breast cancer. Oncologist. 2006;11(8):857-867.

12. National Comprehensive Cancer Network. NCCN Clinical Practice Guideline in Oncology-Breast Cancer, Version 1.2013. 2013. March 15, 2013. Available from: https://www.nccn.org/professionals/physician_gls/ default.aspx. Accessed: July 01, 2018.

13. Moy B, Kirkpatrick P, Kar S, Goss P. Lapatinib. Nat Rev Drug Discov. 2007;6(6):431-432.

14. Cameron D, Casey M, Press M, et al. A phase III randomized comparison of lapatinib plus capecitabine versus capecitabine alone in women with advanced breast cancer that has progressed on trastuzumab: updated efficacy and biomarker analyses. Breast Cancer Res Treat. 2008; 112(3):533-543.

15. Baselga J, Cortés J, Kim SB, et al. Pertuzumab plus trastuzumab plus docetaxel for metastatic breast cancer. N Engl J Med. 2012;366(2): 109-119.
16. de Azambuja E, Holmes AP, Piccart-Gebhart M, et al. Lapatinib with trastuzumab for HER2-positive early breast cancer (NeoALTTO): survival outcomes of a randomised, open-label, multicentre, phase 3 trial and their association with pathological complete response. Lancet Oncol. 2014;15(10):1137-1146.

17. Criscitiello C, Azim HA, de Azambuja E, Rubio IT. Factors affecting surgical management following neoadjuvant therapy in patients with primary HER2-positive breast cancer: results from the NeoALTTO phase III trial. Ann Oncol. 2014;25(4):910-911.

18. Untch M, Loibl S, Bischoff J, et al. Lapatinib versus trastuzumab in combination with neoadjuvant anthracycline-taxane-based chemotherapy (GeparQuinto, GBG 44): a randomised phase 3 trial. Lancet Oncol. 2012;13(2):135-144.

19. Carey LA, Berry DA, Cirrincione CT, et al. Molecular heterogeneity and response to neoadjuvant human epidermal growth factor receptor 2 targeting in CALGB 40601, a randomized Phase III trial of paclitaxel plus trastuzumab with or without lapatinib. J Clin Oncol. 2016; 34(6):542-549.

20. Alba E, Albanell J, De La Haba J, et al. Trastuzumab or lapatinib with standard chemotherapy for HER2-positive breast cancer: results from the GEICAM/2006-14 trial. Br J Cancer. 2014;110(5):1139-1147.

21. Cortazar P, Zhang L, Untch M, et al. Pathological complete response and long-term clinical benefit in breast cancer: the CTNeoBC pooled analysis. Lancet. 2014;384(9938):164-172.

22. Robidoux A, Tang G, Rastogi P, et al. Lapatinib as a component of neoadjuvant therapy for HER2-positive operable breast cancer (NSABP protocol B-41): an open-label, randomised phase 3 trial. Lancet Oncol. 2013;14(12):1183-1192.

23. Guarneri V, Frassoldati A, Bottini A, et al. Preoperative chemotherapy plus trastuzumab, lapatinib, or both in human epidermal growth factor receptor 2-positive operable breast cancer: results of the randomized phase II CHER-LOB study. J Clin Oncol. 2012;30(16):1989-1995.

24. Witzel I, Loibl S, von Minckwitz G, et al. Predictive value of HER2 serum levels in patients treated with lapatinib or trastuzumab - a translational project in the neoadjuvant GeparQuinto trial. Br J Cancer. 2012;107(6):956-960.

25. Baselga J, Bradbury I, Eidtmann H, et al. Lapatinib with trastuzumab for HER2-positive early breast cancer (NeoALTTO): a randomised, openlabel, multicentre, phase 3 trial. Lancet. 2012;379(9816):633-640.

26. Konecny GE, Pegram MD, Venkatesan N, et al. Activity of the dual kinase inhibitor lapatinib (GW572016) against HER-2-overexpressing and trastuzumab-treated breast cancer cells. Cancer Res. 2006;66(3):1630-1639.

27. Xia W, Gerard CM, Liu L, Baudson NM, Ory TL, Spector NL. Combining lapatinib (GW572016), a small molecule inhibitor of ErbB1 and ErbB2 tyrosine kinases, with therapeutic anti-ErbB2 antibodies enhances apoptosis of ErbB2-overexpressing breast cancer cells. Oncogene. 2005;24(41):6213-6221.

28. Vazquez-Martin A, Oliveras-Ferraros C, Cufí S, del Barco S, MartinCastillo B, Menendez JA. Lapatinib, a dual HER1/HER2 tyrosine kinase inhibitor, augments basal cleavage of HER2 extracellular domain (ECD) to inhibit HER2-driven cancer cell growth. J Cell Physiol. 2011;226(1):52-57.

29. Scaltriti M, Verma C, Guzman M, et al. Lapatinib, a HER2 tyrosine kinase inhibitor, induces stabilization and accumulation of HER2 and potentiates trastuzumab-dependent cell cytotoxicity. Oncogene. 2009; 28(6):803-814.

30. Swain SM, Kim SB, Cortés J, et al. Pertuzumab, trastuzumab, and docetaxel for HER2-positive metastatic breast cancer (CLEOPATRA study): overall survival results from a randomised, double-blind, placebo-controlled, phase 3 study. Lancet Oncol. 2013;14(6):461-471.

31. Untch M, Fasching PA, Konecny GE, et al. Pathologic complete response after neoadjuvant chemotherapy plus trastuzumab predicts favorable survival in human epidermal growth factor receptor 2overexpressing breast cancer: results from the TECHNO trial of the AGO and GBG study groups. J Clin Oncol. 2011;29(25):3351-3357.

32. Sliwkowski MX, Lofgren JA, Lewis GD, Hotaling TE, Fendly BM, Fox JA. Nonclinical studies addressing the mechanism of action of trastuzumab (Herceptin). Semin Oncol. 1999;26(4 Suppl 12):60-70. 
33. Ocaña A, Amir E, Pandiella A. Dual targeting of HER2-positive breast cancer with trastuzumab emtansine and pertuzumab: understanding clinical trial results. Oncotarget. 2018;9(61):31915-31919.

34. von Minckwitz G, Procter M, de Azambuja E, et al. Adjuvant pertuzumab and trastuzumab in early HER2-positive breast cancer. $N$ Engl J Med. 2017;377(2):122-131.

35. Murthy RK, Raghavendra AS, Hess KR, et al. Neoadjuvant pertuzumabcontaining regimens improve pathologic complete response rates in stage II to III HER-2/neu-positive breast cancer: a retrospective, single institution experience. Clin Breast Cancer. Epub 2018 Jul 10.
36. Tabernero J, Hoff PM, Shen L, et al. Pertuzumab plus trastuzumab and chemotherapy for HER2-positive metastatic gastric or gastrooesophageal junction cancer (JACOB): final analysis of a double-blind, randomised, placebo-controlled phase 3 study. Lancet Oncol. 2018; 19(10):1372-1384.

\section{Publish your work in this journal}

OncoTargets and Therapy is an international, peer-reviewed, open access journal focusing on the pathological basis of all cancers, potential targets for therapy and treatment protocols employed to improve the management of cancer patients. The journal also focuses on the impact of management programs and new therapeutic agents and protocols on

\section{Dovepress}

patient perspectives such as quality of life, adherence and satisfaction The manuscript management system is completely online and includes a very quick and fair peer-review system, which is all easy to use. Visit http://www.dovepress.com/testimonials.php to read real quotes from published authors.

Submit your manuscript here: http://www.dovepress.com/oncotargets-and-therapy-journal 